\title{
Placenta acreta en el primer trimestre de la gestación. Presentación de dos casos y revisión de la literatura
}

\author{
Lilia M. Sánchez B.1; Edith Angel Muller²; Elvira Castro de Pabón³.
}

\begin{abstract}
RESUMEN
En esta ocasión presentamos dos casos de pacientes con diagnóstico de un acretismo placentario durante el primer trimestre de la gestación, uno de los cuales se manifestó como un síndrome de respuesta inflamatoria sistémica y el otro como una hemorragia uterina anormal. Se han reportado pocos casos de placenta acreta en el primer trimestre de la gestación, algunos de los cuales han requerido histerectomía. El considerar a esta entidad en el diagnóstico diferencial de las hemorragias durante el primer trimestre de gestación podría llevar a un tratamiento más oportuno y a la disminución de sus complicaciones.
\end{abstract}

PALABRAS CLAVES: Placenta acreta, primer trimestre de la gestación, increta, percreta, hemorragia uterina.

\section{SUMMARY}

We report two cases of placenta accrete during the first trimester of pregnancy, one of them presented as a systemic inflammatory response syndrome and the other as an abnormal uterine bleeding. There are few cases reported in the literature of placenta accreta during the first trimester of gestation, some of which required hysterectomy. To consider this entity within the differential diagnosis of uterine bleeding during the first trimester of pregnancy could lead to a prompt treatment diminishing its complications.

KEY WORDS: Placenta accreta, first trimester of gestation, increta, percreta, uterine bleeding.

\section{Introducción}

Aun cuando el acretismo placentario (AP) es una entidad poco frecuente, es una de las principales causas de hemorragia uterina post-parto, que puede llegar a requerir en su manejo una histerectomía obstétrica. Hay pocos casos reportados en la literatura de placenta acreta (PA) en el primer trimestre de la gestación, en esta ocasión presentamos dos, uno de los cuales se manifestó como un síndrome de respuesta inflamatoria sistémica (SRIS) y otro como una hemorragia uterina anormal (HUA)

\section{Presentación de los casos}

\section{Caso 1}

Paciente de 34 años G5P3A2, con FUR 10-11-99, a quién se le realizó un primer legrado el 27-12-99 por aborto incompleto y un segundo legrado el 20-01-00 por retención de restos, en esta ocasión presentó además fiebre por lo que le iniciaron antibióticos (A/B) y el 26-01-00 fue remitida al Instituto Materno Infantil (IMI) con diagnóstico de infección posaborto. Tenía una bhCG del 10-01-00 en 28.200 $\mathrm{mUl} / \mathrm{ml}$. Se hospitalizó con impresión diagnóstica (ID) de enfermedad pélvicainfIamatoria (EPI) pos-legrado parcialmente tratada y enfermedad trofoblástica gestacional (ETG), sin embargo, la bHCG de control fue de $850 \mathrm{mUl} / \mathrm{ml}$, no se encontraron signos de infección y la ecografía pélvica mostró una imagen sugestiva de retención de restos. Se decidió realizar un nuevo legrado obstétrico y se obtuvo escaso material de aspecto ovular no fétido. No se administraron A/B y la paciente evolucionó en forma satisfactoria.

Reingresó 10 días después por cuadro de 1 día de evolución de fiebre, escalofrío, malestar general, disnea y dolor en hipogástrio y hemitórax derecho. Al examen se encontró: TA 90/60, FC 110x', T $38^{\circ} \mathrm{C}$, S02 87\%. C/P: RsCs taquicárdicos y movilización de secreciones. Dolor en el hipogastrio a la palpación profunda, sin peritonismo. G/U: vagina hipertérmica, sin sangrado, útero en RVF, tamaño normal, dolor a la movilización cervical y a la

Profesora Asistente. Departamento de Patología. Facultad de Medicina. Universidad Nacional de Colombia. Instituto Materno Infantil. Profesora Asistente. Departamento de Ginecología y Obstetricia. Facultad de Medicina. Universidad Nacional de Colombia. Instituto Materno Infantil. Profesora Asistente. Departamento de Patología. Facultad de Medicina. Universidad Nacional de Colombia. Instituto Nacional de Cancerología 


\section{Foto 1 \\ CORTE TRANSVERSAL DE LA PARED MIOMETRIAL DONDE SE OBSERVA SOLUCIÓN DE CONTINUIDAD. EN LA HISTOLOGÍA ESTA ZONA CORRESPONDE A LA PLACENTA INCRETA}

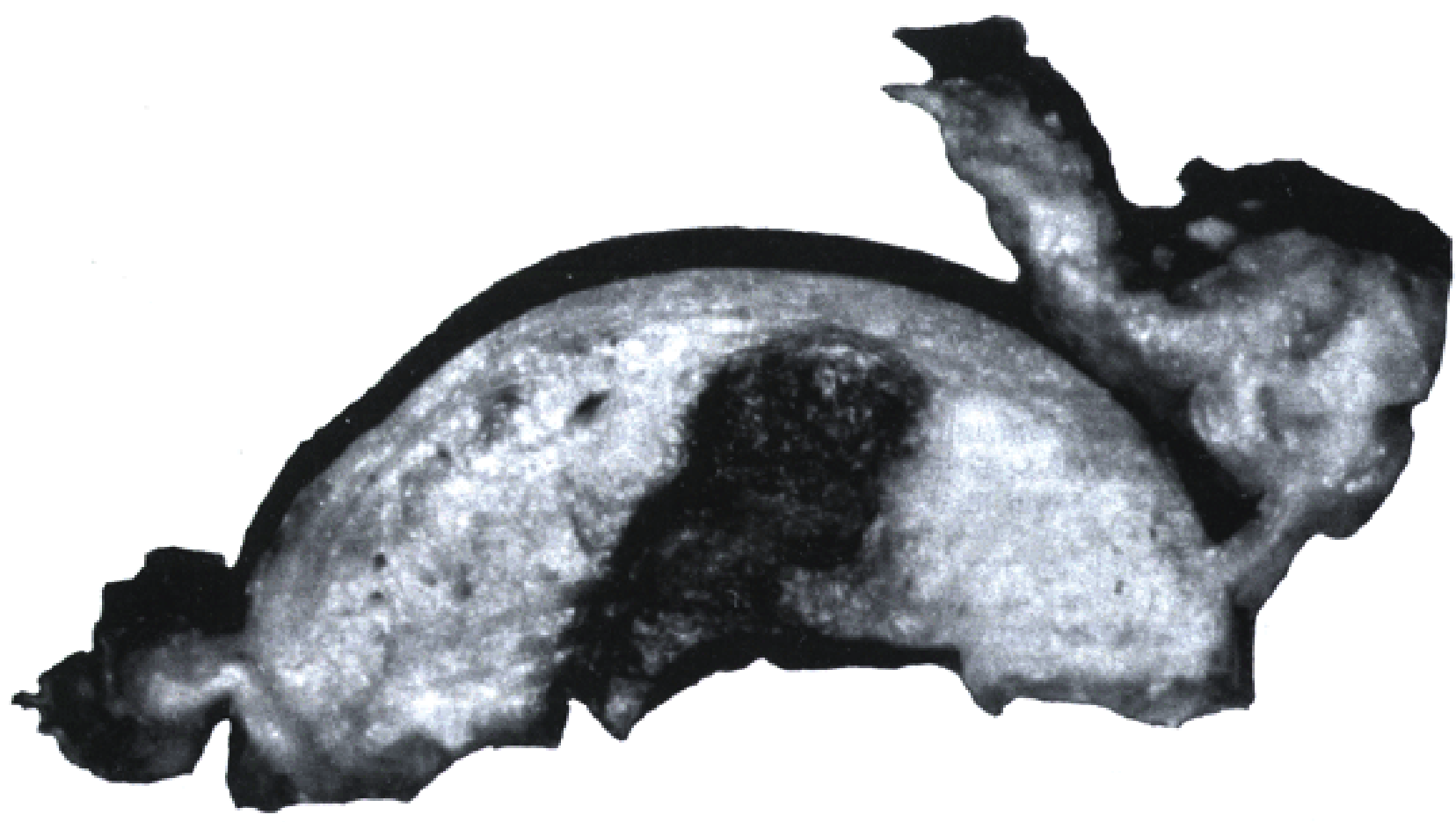

palpación del anexo derecho, no masas y fondos de saco libres. Se realizó ID de EPI post legrado, sepsis y bronconeumonía basal derecha. Se inició manejo con A / B (Clindamicina-gentamicina). Al día siguiente persistía con picos febriles y disnea. Los laboratorios mostraron $\mathrm{CH}: 8500$ PMN: 84\%, Hb: 9mg/dL, plaquetas: 83.000/ $\mathrm{mm}^{3}$, creatinina $0.89 \mathrm{mg} / \mathrm{dL}$. Gases arteriales: acidosis metabólica. La ecografía pélvica, mostró en la cavidad endometrial una imagen de ecogenicidad mixta de $2.6 \times 1.8 \times 1.6 \mathrm{~cm}$, con ID de retención de restos. La paciente persistió hipotensa, taquicárdica y disneica. Se inició monitorización invasiva, soporte inotrópico y se cambiaron $\operatorname{los} \mathrm{A} / \mathrm{B}$ a Clindamicina-Amikacina. Al otro día se encontró leucocitosis. El informe de patología del legrado anterior fue deciduo-endometritis severa y cambios sugestivos de mola completa. Al tercer día de hospitalización persistió con picos febriles, sin mejoría en los signos vitales, por lo cual se pasó a laparotomía con ID de sepsis secundaria a miometritis, encontrándose un útero de tamaño, forma y consistencia normal, al igual que los anexos y los infundíbulos pélvicos. Se realizó histerectomía total abdominal y revisión de infundíbulos pélvicos. En el post-operatorio presentó una atelectasia bilateral que se manejó con terapia respiratoria evolucionando satisfactoriamente, se le dio salida el 15-02-00.

Estudio anatomopatológico: $\mathrm{Se}$ recibió un útero desprovisto de anexos que pesó $80 \mathrm{~g}$ y midió $6 \mathrm{~cm}$ entre los cuernos y $8 \mathrm{~cm}$ del fondo al exocervix. La cavidad endometrial estaba ocupada por coágulos. A los cortes seriados el miometrio tenía un espesor de $1.5 \mathrm{~cm}$ y el endometrio de $0.2 \mathrm{~cm}$. En la pared anterior en la proximidad al istmo se evidenció una solución de continuidad ocupada por sangre que medió en profundidad $1.3 \mathrm{~cm}$ y en la base $1 \mathrm{~cm}$, llegando a $25 \mathrm{~mm}$ de la serosa (Foto 1). El estudio microscópico de esta zona mostró la presencia de vellosidades coriales desvitalizadas y edematosas, sin proliferación anormal del trofoblasto, mezcladas con sangre y fibrina que infiltraban la pared miometrial casi hasta la serosa. Además se encontraron focos de Adenomiosis. El diagnóstico final en este caso fue el de una Placenta Increta en un útero que se encontraba en el primer trimestre de la gestación.

\section{Caso 2}

Paciente de 39 años, G5P4A1, ingresó al IMI, el 3-0200, por menometrorragia asociada a dolor hipogástrico, 
astenia y adinamia. 6 meses antes había tenido sangrado genital abundante que fue interpretado como aborto incompleto. Le realizaron legrado y el informe de patología, según la paciente, fue "Mola". No tuvo controles ni seguimiento de bhCG. La paciente no planificaba. Se encontró una paciente en regular estado general, con palidez mucocutánea, TA: 120/70, FC: 94/min, FR: 201 min. Dolor a la palpación profunda en hipogastrio, sin masas ni signos de irritación peritoneal. Sangrado genital, útero en AVF aumentado de tamaño, de aproximadamente $14 \mathrm{~cm}$, doloroso a la palpación, reblandecido, parametrios normales y fondos de saco libres. La ID fue HUA, ETG y síndrome anémico. La radiografía de tórax fue normal, la ecografía pélvica mostró un útero de $13 \mathrm{~cm}$, con una cavidad endometrial ocupada por una masa fúndica, hacia la pared anterior de 5 x 4 x 4,5cm compatible con mioma submucoso Vs mola. $\mathrm{CH}$ : sin leucocitosis, $\mathrm{Hb}: 7.8 \mathrm{mg} / \mathrm{dl}$ y prueba de embarazo positiva. Se transfundió 1U. de glóbulos rojos empaquetados. A los tres días, presentó emésis en 2 ocasiones y dolor abdominal intenso, en hipocondrio derecho y epigastrio, asociado a sangrado genital. Se encontró álgica, con TA: 90/60, FR: 24/min, FC: 96/min, hipoventilación pulmonar, aumento del perímetro abdominal y signos de irritación peritoneal. Se interrogó posible cuadro de colecistitis-colelitiasis y se realizó ecografía abdominal que mostró abundante líquido libre en cavidad y órganos abdominales normales. Posteriormente se encontró ingurgitación yugular, estertores basales en ambos campos pulmonares, abdomen distendido con francos signos de irritación peritoneal y T.V. con abombamiento del fondo de saco posterior, extremidades edema GIl. Se realizó ID de abdomen agudo, insuficiencia cardíaca congestiva y perforación uterina Vs. coriocarcinoma. Se inició monitorización invasiva con catéter central y apoyo inotrópico. Se realizó laparotomía exploratoria encontrándose ascitis $1500 \mathrm{cc}$, estructuras viscerales normales, útero de $15 \mathrm{~cm}$ reblandecido en cara anterior y fondo, anexos e infundíbulos pélvicos normales, a la manipulación del útero presentó sangrado vaginal abundante, no fétido. Se tomó muestra de líquido peritoneal y con diagnóstico de ETG vs. miometritis, se realizó histerectomía total abdominal.

En postoperatorio inmediato, requirió transfusión de 2 U. de glóbulos rojos, se continuó apoyo inotrópico y monitorización permanente. La evolución

Foto 2

CORTE TRANSVERSAL DEL ENDOMIOMETRIO, SOBRE LA SUPERFICIE ENDOMETRIAL SE EVIDENCIA ADHERIDO UN TEJIDO DE ASPECTO VELLOSO

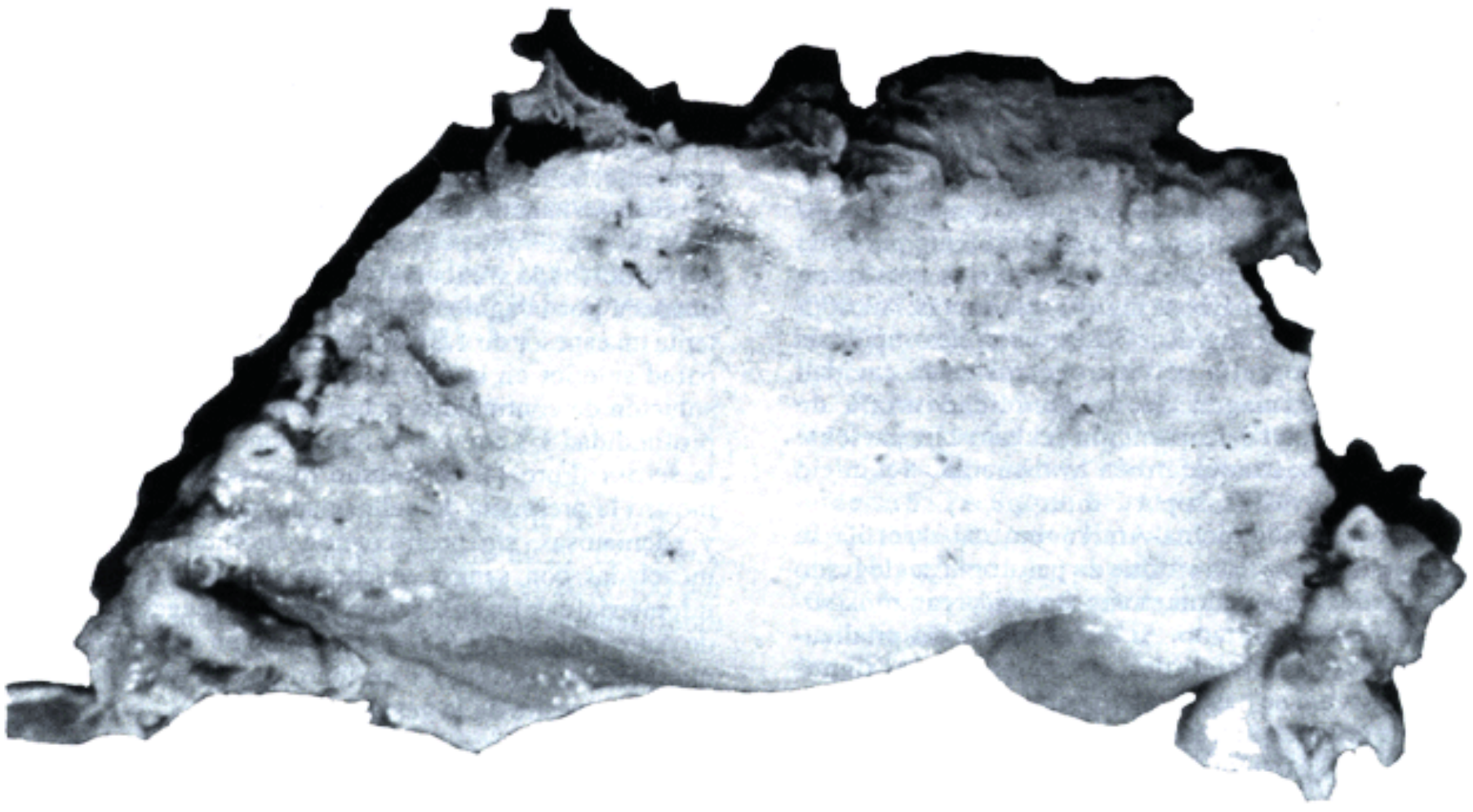


de la paciente fue satisfactoria $y$ se dio de alta el día 13-02-00.

Estudio anatomopatológico: Se recibió un útero sin anexos que pesó 180 g y midió $4 \mathrm{~cm}$ entre los cuernos y $10 \mathrm{~cm}$ del fondo al exocervix. La cavidad endometrial estaba ocupada en un $40 \%$ por una lesión elevada que midió 4 x 4.5 $\mathrm{cm}$, de aspecto velloso y bordes bien definidos de 0.5 cm de espesor, esta lesión surgía a partir de la superficie endometrial en el fondo uterino $\mathrm{y}$ las paredes anterior $\mathrm{y}$ posterior. A los cortes seriados el miometrio tenía un espesor de $3 \mathrm{~cm}$ (Foto 2). El estudio microscópico de esta lesión mostró la presencia de vellosidades coriales inmaduras algunas des vital izadas y edematosas, sin proliferación anormal del trofoblasto, mezcladas con sangre y fibrina, en contacto directo con el miometrio. La conclusión en este caso fue la de una PA en un útero que se encontraba en el primer trimestre de la gestación. Además se encontraron focos de Adenomiosis y Miometritis subaguda.

\section{Discusión}

Se denomina a la placenta como acreta cuando ésta se implanta en zonas donde la decidua es deficiente o anormal y por tanto hay una infiltración del miometrio por vellosidades coriales; esta infiltración puede ser focal, parcial o total. A su vez esta condición se subdivide en acreta, increta y percreta. La placenta increta y percreta infiltran todo el espesor de la pared miometrial, en la percreta además las vellosidades perforan la serosa y llegan en algunas ocasiones a infiltrar órganos vecinos, especialmente la vejiga. La PA está limitada a la superficie miometrial. La frecuencia de presentación del AP varía entre 10 y 48 por 10.000 partos. (Oishi A 1999, Hung TH 1999, Zaki ZM, 1998).

En las mujeres con acretismo placentario se han visto factores de riesgo, dentro de los cuales se encuentran:

1. Edad y multiparidad: la presentación de AP, aumenta con la paridad de la paciente y la edad, siendo muy rara en primíparas.

2. Placenta previa: esta se ha encontrado en el $30 \%$ de los casos de PA. Igualmente se ha visto PA en el 9,3\% de las pacientes con placenta previa (Miller DA, 1997).

3. Cesárea anterior, o cirugías uterinas previas: se ha visto este antecedente en el $25 \%$ de los casos. En el $29 \%$ de los casos la PA estaba implantada en la cicatriz uterina y solo en el $5 \%$ la placenta estaba implantada en otro sitio. (Miller DA, 1997).

4. Dilatación y legrado, en el $25 \%$ de los casos.

5. Infección uterina previa, remoción manual de la placenta, leiomiomas y otras anomalías uterinas: la asociación con estas entidades es inconstante.

6. Niveles anormalmente elevados de a feto-proteína y de b-HCG, en el segundo trimestre. (Hung TH, 1999). Aunque su etiología permanece en debate, se considera que para el desarrollo de esta entidad debe existir una deficiencia focal o difusa de la decidua que permite que las vellosidades coriales y el fibrinode del plato basal se adhieran directamente al miometrio adyacente. En el lugar de la adhesión anormal de la placenta la decidua no es aparente y hay un exceso de células del citotrofoblasto (CCT), este último es hallazgo normal en el plato basal solo al final del embarazo.

La decidua se considera como una barrera que previene la invasión del trofoblasto. En ella se encuentra una glicoproteína fibrinoide que reviste las células de la decidua protegiendo a las células del trofoblasto de un ataque inmunológico. Al parecer el efecto de barrera de la decidua sería temporal, pues se sabe hay un paso temprano en el embarazo de CCT a través de la decidua para alcanzar el miometrio. Sin embargo, esto no significa que la decidua en estos casos tenga que estar ausente desde el inicio de la gestación. La placentación hemocorial requiere la formación de decidua, no obstante durante la placentación hay necrosis y destrucción de la misma, por lo que parece existe un balance entre la decidua y el trofoblasto invasor, en efecto la decidua parece ser una limitante temporal mas que un tejido obstructivo.

Es más probable que después de una nidación normal en el endometrio, el lecho de la placenta se expanda pudiendo llegar hasta zonas del endometrio con cicatrices, o al segmento inferior $y$ al istmo o a la parte superior del canal endocervical donde la decidualización es menos adecuada. El trofoblasto no velloso invasor sobrepasa la mucosa pobremente decidualizada para permitir la penetración profunda. Esto podría ser válido en las alteraciones focales y la placenta previa.

El hecho de ser más frecuente en múltiparas sugiere que el balance entre el trofoblasto invasor y la acción represora de la decidua cambia progresivamente a favor del primero, además se ha observado que el antecedente de extracción manual de la placenta en partos anteriores, es frecuente en estas pacientes.

La penetración en la profundidad de las vellosidades se acompaña de una extensión de la migración fisiológica del trofoblasto endovascular a las arterias de gran calibre, como las arterias radiadas, lo cual puede explicar el sangrado en los casos de AP en una placenta previa. Mientras que las arterias espiraladas superficiales y aun algunas arterias profundas, en estrecho contacto con el trofoblasto, no sufren cambios fisiológicos. Se ha visto además que no hay disminución aparente de la decidua parietal en el AP.

El trofoblasto extravelloso presente es uni o binuclear con escasez de las células sincitiales gigantes presentes en la placentación normal. Hay una aparente proliferación del trofoblasto intersticial en la unión de la placenta con el miometrio, pero la densidad del trofoblasto intersticial en la profundidad del miometrio es menor que en la placentación normal. Estos hallazgos sugieren que existe un defecto en la interacción entre los tejidos maternos, particularmente la decidua y el trofoblasto migratorio, en los estadios tempranos de la placentación. Resultando en una adhesión inadecuada de la placenta o en la penetración, y en el desarrollo anormal de la circulación uteroplacentaria. Esta hipótesis se basa en que este trastorno es raro antes de la semana 20 y en el hallazgo incidental, que se vio, en un útero con un embrión de $2 \mathrm{~cm}$, donde se encontró unión directa de las vellosidades a la musculatura uterina, en el sitio de implantación. 
Una placenta adherente o penetrante no es fácil de diagnosticar antes del alumbramiento. Después de éste, se manifiesta como retención placentaria y sangrado uterino. El diagnóstico generalmente se realiza, después de intentar la extracción manual de la placenta. La morbilidad y la mortalidad maternas están significativamente aumentadas en la placenta percreta. La mortalidad secundaria a la hemorragia y sus complicaciones puede ser hasta del 10\% (Cantanzarite V, 1996). La pérdida masiva de sangre intraoperatoria puede necesitar transfusión masiva de sangre con el riesgo inherente de coaguláción intravascular diseminada, reacciones a la transfusión, sobrecarga de líquidos e infección. La morbilidad quirúrgica de la histerectomía incluye la lesión de intestino y lesiones urológicas principalmente el trauma ureteral y las lesiones de vejiga (Hudon L, 1998)

Antes de 1990 habían sido reportados 8 casos de AP postaborto, de los cuales 4 se manifestaron con ruptura espontánea del útero, 3 durante el curetaje y 1 fue un hallazgo incidental, en estos el factor de riesgo más importante que se encontró fue el antecedente de trauma uterino, ese mismo año se informa el caso de una PA del primer trimestre la cual se encontró durante un curetaje-succión por aborto (Harden MA, 1990). Posteriormente, ha habido reportes aislados, como el informe en 1992 de una placenta increta, que complicó un aborto del primer trimestre, este AP fue visto en una mujer de 32 años G6 con antecedente de placenta acreta y cesárea por hipertensión asociada al embarazo (Ecker JL, 1992) Y en 1996 la presentación de una placenta increta en un embarazo de menos de 20 semanas con huevo anem-brionado que requirió la Histerectomia para el control de la hemorragia (Gist RS, 1996). Otra paciente que presentó un AP en la semana 25 de gestación se manifestó como una ruptura espontánea en el fondo del útero, la cual requirió histerectomía, en este caso el estudio anatomopatológico mostró una placenta percreta (Kinoshita T, 1996).

Actualmente la ecografía y el Doppler color al igual que la resonancia magnética nuclear pueden ayudar en el diagnóstico del AP. Con estos métodos, se pueden ver la invasión placentaria al miometrio, e incluso a órganos vecinos. (Kirkinen $\mathrm{P}, 1998)$

El ultrasonido es útil en el tamizaje del AP, pero requiere una evaluación cuidadosa de la interfase uteroplacentaria. Algunos autores recomiendan la vía abdominal con vejiga distendida. Otros prefieren la via transvaginal con vejiga vacía o semidistendida. Los criterios ecográficos descritos para el diagnóstico de AP son (Hudon L, 1998):
1. Ausencia o adelgazamiento (menos de $1 \mathrm{~mm}$ ) de la zona miometrial hipoecoica normal en el segmento inferior anterior.

2. Presencia de espacios vasculares lacunares dentro del parénquima placentario, lo que se ha llamado "apariencia de queso suizo".

3. Adelgazamiento, irregularidad o disrupción focal de la interfase hiperecoica entre las paredes uterina y vesical.

4. Extensión del tejido placentario mas allá de la serosa uterina.

5. Identificación con Doppler de vasos placentarios que se extiendan al miometrio o a la vejiga.

Algunos de los casos de AP vistos en la primera mitad
del embarazo han sido diagnosticados mediante imagenología, mientras que otros se han diagnosticado solamente cuando presentan complicaciones, como ruptura uterina, persistencia de sangrado uterino y/o necesidad de legrado uterino (Wheeler TC, 1996, ArredondoSoberon F, 1995, Rashbaum WK, 1995). En Brasil reportaron un caso de placenta percreta diagnosticada por ultrasonido y Doppler color en la semana catorce de gestación; en este caso se realizó un manejo inicial fallido con metrotexate IM, seguido por histerectomía total, durante la cual se observó ruptura del útero, con infiltración de la placenta a la pared posterior de la vejiga. (Passini Junior R, 1996).

La prevención de las complicaciones del AP se basa en el reconocimiento temprano de los factores de riesgo, y la realización del diagnóstico preparto con la ayuda de la imagenología. Más de un $80 \%$ de las pacientes con un diagnóstico ecográfico de placenta percreta requerirán una cesárea-histerectomía (Finberg G, 1992). Se necesita un grado alto de sospecha y preparación para disminuir la morbilidad, mediante la prevención de las complicaciones médicas y quirúrgicas. El manejo óptimo involucra la participación, al lado del obstetra, de un equipo multidisciplinario que incluya radiología, anestesia, urología, hematología, banco de sangre, cirugía y neonatología.

La incidencia de AP está aumentando. Esto se debe al aumento en el número de pacientes con cesáreas múltiples. El examen con ultrasonido tiene un valor predictivo positivo del $80 \%$ para el diagnóstico de placenta acreta/ percreta. La placenta acreta/percreta la podemos encontrar en primera mitad de la gestación, como se muestra en este artículo, por lo tanto es un diagnóstico más a tener en mente.

\section{BIBLIOGRAFIA}

Arredondo-Soberon F. Sabella V. Garza-Leal J. Valente PT. Placenta increta en el primer trimestre de embarazo. Ginecol Obstet Mex 1995; 63: 279- 81.

Cantanzarite V, Stanco L, Schrirnmer S et al. Managing placenta previa! accreta. Contemp Obstet Gynecol 1996; 41: 66-95.

Ecker JL, Sorem KA, Soodak L, et al. Placenta Increta Complicating a FirstTrimester Abortion A case report. Journal Reproductive Medicine.

$1992 ; 37: 10$
Finberg G, Williams J. Placenta accreta: prospective sonographics diagnosis in patients with placenta previa/accreta. Contemp Obstet Gynecol 1996; 41: 66-95.

Gist RS, Vuong V, Brody S, Rees P, Landry AD. Placenta increta occurring in a blighted ovum. South Med J. 1996; 89(5): 545-7.

Harden, MA, Walters MD, Valente PT. Postabortal hemorrahage due to placenta increta: A case report. Obstet Gynecol. 1990; 75: 523. 
Hudon L, Belfort MA, Broome DR. Dosis and management of placenta percreta: A review. Obstet Gynecol survey 1998; 53: 509-517.

Hung TH, Shau WY, Hsieh CC, et al. Risk factors for placenta accreta. Obstet Gynecol 1999; 93: 545-50.

Kinoshita T, Ogawa K, Yasumizu T, Kato J. Spontaneous rupture of the uterus due to placenta percreta at 25-weeks' gestation : a case report. J Obstet Gynaecol Res 1996; 22: 125-8.

Kirkinen P, Helin-Martikainen HL, Vanninen R, Partanen K. Placenta accreta: imaging by gray-scaleand contrast enhanced color Doppler sonography and magnetic resonance imaging. J Clin Ulttasound 1998; 26: 90-4.

Miller DA, Chollet JA, Goodwin TM. Clinical risk factors for placenta previa-placenta accreta. Am J Obstet Gynecol 1997; 177: $210-4$.

Oishi A, Yasuda S, lnaba N. Placenta accreta/increta. Review of 10 cases and a case report. Arch Gynecol Obstet 1999; 263: 69-72.
Passini-Junior R, Knobel R, Barini R, Marussi E. Placenta percreta with silent rupture of the uterus. Rev Paul Med 1996; 114: 1270-3.

Rashbaum WK, Gates EJ, Jones J et al. Placenta accreta encountered during dilatation and evacuation in the second trimester. Obstet Gynecol 1995; 85: 701-3.

Wells M, Mohamdee O. Patology of the pregnant uterus. In Haines \& Taylor Obstetrical and Gynecological Pathology, Edited by Fox H. Churchill Livingstone. Fourth edition Vol. 2 1995; 1529-32.

Wheeler TC, Anderson TL, Kelly J, Bochm FH. Placenta previa increta diagnosed at 18-weeks' gestation. J Reprod Med 1996; 41: 198200.

Zaki ZM, Bahar AM, Ali ME et al. Risk factors and morbidity in patients with placenta previa accreta compared to placenta previa nonaccreta. Acta Obstet Gynecol Scand 1998; 77: 391-4. 